ISSN 0103-9954

\title{
TAXA DE CORTE SUSTENTÁVEL PARA MANEJO DAS FLORESTAS TROPICAIS
}

\author{
SUSTAINABLE ALLOWABLE CUT FOR TROPICAL FOREST MANAGEMENT
}

Evaldo Muñoz Braz ${ }^{1}$ Paulo Renato Schneider ${ }^{2}$ Patricia Povoa de Mattos ${ }^{3}$

Gerson Luiz Selle ${ }^{4}$ Fabio Thaines ${ }^{5}$ Luciano Arruda Ribas ${ }^{6}$ Elisabete Vuaden ${ }^{7}$

\section{RESUMO}

Existe uma grande lacuna dos planos de manejo de florestas tropicais com relação à intensidade de extração e às taxas de corte, usualmente sendo definidas de forma arbitrária. Este trabalho visa definir intensidades de corte diferenciadas para grupos de espécies arbóreas comerciais, com diferentes ritmos de crescimento, por unidade de produção, no estado do Amazonas. Utilizou-se o incremento periódico anual percentual em volume, de 26 espécies arbóreas, obtido de parcelas permanentes. O incremento periódico anual, percentual em volume, para efeito diferenciador, considerou $1 \%$ como diferença limite. Foram identificadas três intensidades de corte, para as classes comerciais: 24,4\% (grupo I), 35,4\% (grupo II) e 42,4\% (grupo III). Considerando a exploração total sustentável por hectare, para as 26 espécies, seria de $11,5 \mathrm{~m}^{3} \mathrm{ha}^{-1}$ com intensidade de corte de $37 \%$. O procedimento de cálculo é simples e pode ser utilizado pelos órgãos fiscalizadores, certificadores ou legisladores como balizador do ciclo de corte e taxa de corte.

Palavras-chave: manejo florestal; incremento periódico anual; intensidade de corte; sustentabilidade.

\begin{abstract}
There is a large gap in tropical forest management plans regarding to the intensity of logging and cutting rates. So far, it has been defined arbitrarily. This work aims at to define different cutting intensities for groups of tree species with different growth rhythm, in the state of Amazonas, Brazil. The study used the periodical annual increment, percentual in volume, of 26 species, obtained from permanent plots. The periodic increment percentage in volume for differentiating effect considered $1 \%$ difference as threshold. Three levels of logging intensity were identified for the commercial classes: $24.4 \%$ for group I, $35.4 \%$ for group II, and $42.4 \%$ for group III. The total sustainable cutting rate was $11.5 \mathrm{~m}^{3} \mathrm{ha}^{-1}$ with cut intensity of $37 \%$. The cutting rate calculation procedure is very simple and can be used by forest law and monitoring institutions.
\end{abstract}

Keywords: forest management; periodical annual increment; cut intensity; sustentability.

1. Engenheiro Florestal, Dr., Pesquisador da EMBRAPA Florestas, Caixa Postal 319, CEP 83411-000, Colombo, (PR).evaldo@cnpf.embrapa.br

2. Engenhario Florestal, Dr., Professor Titular do Departamento de Ciências Florestais, Centro de Ciências Rurais, Universidade Federal de Santa Maria, Av. Roraima, 1000, CEP 97105-900, Santa Maria (RS). paulors@smail.ufsm.br

3. Engenheira Agrônoma, Dr ${ }^{\mathrm{a}}$, Pesquisadora da EMBRAPA Florestas, Caixa Postal 319, CEP 83411-000, Colombo (PR).povoa@cnpf.embrapa.br.

4. Engenheiro Florestal, Dr., Servidor Público Federal do Departamento de Ciências Florestais, Centro de Ciências Rurais, Universidade Federal de Santa Maria, Av. Roraima, 1000, CEP 97105-900, Santa Maria (RS). gersonluizselle@ufsm.br

5. Engenheiro Florestal, Coodenador da TECMAN - Tecnologia e Manejo Florestal, CEP 69908-970, Rio Branco (AC). Colabora com projetos da Embrapa Acre. fabiothaines@tecman.eng.br

6. Engenheiro Florestal, Dr., Pesquisador da EMBRAPA Acre, Caixa Postal 321, CEP 69908-970, Rio Branco (AC). laribas@cpafac.embrapa.br

7. Engenheira Florestal, MSc., Professora da Universidade Tecnológica Federal do Paraná, Estrada para Boa Esperança, Km 04, CEP 85660-000, Dois Vizinhos (PR). elisabetevuaden@yahoo.com.br

Recebido para publicação em 6/12/2010 e aceito em 24/03/2011 


\section{INTRODUÇÃO}

Uma das grandes lacunas do manejo reside na quantidade que pode ser extraída da floresta, de maneira sustentável. Para Borsoi (2004) o uso inadequado das florestas naturais pode desequilibrar os ciclos de extração e degradar os ecossistemas.

Nos últimos anos, tem-se privilegiado o conceito de exploração de impacto reduzido, pois extrações elevadas causam um dano substancial ao povoamento, reduzindo sua capacidade produtiva. Entretanto, um ponto importante tem sido desconsiderado: a compatibilização do ciclo de corte com o ritmo de crescimento das espécies "manejadas". Assim, no manejo das florestas naturais, a taxa de extração anual raras vezes é fundamentada na associação de dados sobre a estrutura da floresta e o ritmo de crescimento das espécies, ou seja, os volumes determinados para extração são arbitrários (PUTZ et al., 2000).

O conceito "manejo de florestas" não estará completo, em qualquer plano de manejo, se não contiver uma previsão razoável de quanto a floresta pode recuperar dentro de um intervalo de tempo (BRAZ, 2010).

Do ponto de vista da legislação, no Brasil, a questão tende a ser tratada de maneira simplificada, como o estabelecimento de taxas de corte fixas, independentemente da floresta manejada (BRASIL, 2008). Com isso, tem-se estimulado, indiretamente, a formação de instrumentos apenas burocráticos, em detrimento do uso da técnica para se aprovar, monitorar ou analisar planos de manejo florestal. Equivocadamente, tem-se considerado que apenas baixas taxas de extração podem dar garantia da sustentabilidade da floresta, esquecendo-se que estas devem estar vinculadas, no mínimo, à estrutura das classes diamétricas e à disponibilidade das espécies que comporão essa taxa de corte.

Com as responsabilidades ambientais, cada vez mais definidas nos dias atuais, e com a disponibilidade cada vez maior de profissionais da área florestal, é inexplicável a busca de simplificações sem amparo técnico-científico. A questão não é complicar, mas se amparar em métodos ou ferramentas, já existentes.

A justificativa de que nada se sabe sobre as espécies de interesse comercial já não é argumento aceitável. Existem muitos estudos publicados, baseados em parcelas permanentes, que informam o crescimento de grupos ecológicos de várias espécies das florestas tropicais e estas informações, levantadas por instituições de pesquisa e universidades, podem servir para a elaboração de planos de manejo.

Em muitos casos, para reduzir a variabilidade das informações, é sugerido o agrupamento das espécies que tenham características semelhantes com o ritmo e a distribuição do crescimento (DAWKINS, 1958; ALDER, 1983; VANCLAY, 1994; SÁNCHEZ, 1998).

Alder e Silva (2001), em simulação de exploração florestal, utilizando o CAFOGROMCPATU, enfatizamanão sustentabilidade para retiradas não controladas de todas as árvores comerciais e, mesmo com a inclusão das espécies potenciais, houve decréscimo da produtividade. Isso significa que a não sustentabilidade dos ciclos subsequentes depende não apenas de retiradas altas, mas também de retiradas que desconsideram a estrutura final das árvores pós-corte, não somente das de estoque, como também a estrutura remanescente das árvores das classes comerciais.

Para Schneider e Finger (2000) é necessário que a floresta tropical possa repor o volume de corte, durante o período de tempo definido pelo ciclo de corte estabelecido, garantindo a sustentabilidade de produção da floresta.

Essas observações reforçam a importância da definição de taxas de corte calculadas e distribuídas sobre estruturas previamente determinadas, buscando a regulação da floresta e direcionando a ação, no sentido de otimizar a estrutura remanescente com o incremento desejado.

Além disso, existe a necessidade de métodos e sistemas que garantam a sustentabilidade dos planos de manejo e sejam de fácil utilização, pelas empresas florestais e órgãos fiscalizadores das florestas tropicais (BRIENEN e ZUIDEMA, 2006).

O objetivo deste trabalho é definir intensidades de cortes diferenciadas para grupos de espécies comerciais de unidade de produção, no estado do Amazonas, utilizando sistemas de fácil execução e assimilação por órgãos fiscalizadores e empresas florestais.

\section{MATERIAL E MÉTODO}

$\mathrm{O}$ estudo foi realizado em uma área da empresa ST Manejo de Florestas, denominada compartimento Iracema II, situada no estado do Amazonas, próxima à divisa com os estados do Acre e Rondônia, cuja localização fica entre as coordenadas de $66^{\circ} 30^{\prime \prime}-67^{\circ} \mathrm{W}$ e $9^{\circ}-10^{\circ} 30^{\prime \prime} \mathrm{S}$.

A área total da propriedade é 4.211,67 ha, dos quais 2.000 ha destinam-se ao manejo. $\mathrm{O}$ relevo 
é suave ondulado, com algumas áreas com inclinação forte. A área apresenta cursos d'água apenas temporários, com a floresta sendo predominantemente densa e árvores emergentes, mas ocorrendo também tipologias de floresta aberta com bambu e palmeiras. O solo é Podzólico Vermelho-amarelo distrófico, com pH entre 3,5 e 5,0 (OLIVEIRA et al., 2006).

O clima é do tipo Am (classificação de Köppen), clima quente e úmido de monções, com estação seca bem definida entre os meses de junho e outubro, seguido de estação chuvosa, com temperatura média anual de $25^{\circ} \mathrm{C}$, umidade relativa do ar de $85 \%$ e precipitação anual de $2.250 \mathrm{~mm}$ (OLIVEIRA et al., 2006).

Foi avaliado o volume comercial das espécies de interesse em uma unidade de produção de 547 hectares, mediante censo florestal. Também se avaliou o incremento em volume no período considerado e transformado em valor percentual, obtendo-se assim o incremento periódico anual percentual em volume (IPAv\%), sendo então correspondente às espécies de interesse, identificadas pelos valores médios alcançados em 20 parcelas permanentes (PP) instaladas na área, obtidos no período de 5 anos. As parcelas possuem 1 ha $(100 \mathrm{~m}$ x $100 \mathrm{~m}) \mathrm{em}$ um total de 20 ha. O incremento periódico anual percentual em volume foi obtido pelas fórmulas:

$$
I P A v \%=\left(\frac{I P}{V 1}\right) \frac{100}{n}
$$

Em que: IP $=$ V2 - V1; IPAv $\%=$ Incremento periódico anual percentual em volume; $\mathrm{n}=$ número de anos do período; $\mathrm{IP}=$ incremento periódico; $\mathrm{V} 1=$ volume inicial; $\mathrm{V} 2$ = volume final.

As espécies foram agrupadas de acordo com o ritmo semelhante de IPAv\%. O intervalo entre as classes de incremento ficou estabelecido em $1 \%$.

Depois do agrupamento das espécies pela semelhança do IPA percentual em volume, foram ajustadas curvas de IPAv\% por grupo segundo a equação logarítimica, definida por Schneider e Finger (2000), assim descrita:

$$
\ln I P A v \%=b_{0}+b_{1}(\ln d)
$$

Em que: $\mathrm{d}=$ centro de classe de DAP; $\ln =$ logarítmo neperiano.

A partir do IPAv\% ajustado, foi determinado o IPAv\% médio de cada grupo, através da ponderação pelo número de árvores de cada.

Para cada grupo, foi determinada a intensidade de corte permitida, sendo esta baseada no que a floresta pode incrementar no ciclo considerado. Assim, é calculado o percentual que a floresta tem possibilidade de incrementar durante o período, de forma cumulativa. A intensidade de corte deve ser igual ou menor que a capacidade de incremento das espécies consideradas, sendo o ciclo de corte fixado em 25 anos e a intensidade de corte aplicada sobre as classes comerciais, obtida pela expressão (SCHNEIDER, 1993):

$$
I C=\left\{1-\left(\frac{1}{1,0 i^{c c}}\right)\right\} 100
$$

Em que: $\mathrm{IC}=$ intensidade de corte, em percentagem do volume; $\mathrm{i}=$ incremento periódico anual, percentual em volume; $\mathrm{cc}=$ ciclo de corte em anos.

Foi identificado o volume comercial de cada grupo de espécies, em cada unidade de produção e calculada a taxa de corte pela fórmula:

$$
T C=\frac{V c * I C}{100}
$$

Em que: $\mathrm{TC}=$ taxa de corte em volume $\left(\mathrm{m}^{3}\right)$ para o ciclo de corte; $\mathrm{Vc}=$ volume comercial da floresta disponível, em metros cúbicos.

Para calcular o volume, foi ajustada uma equação para a floresta em estudo, derivada do modelo de Hohenadl-Krenn (FINGER, 1992) e baseada na cubagem na altura comercial $\left(\mathrm{R}^{2}=0,81 ; \mathrm{CV} \%=12,2 \mathrm{e}\right.$ Syx $=0,4040)$ :

$V C=-1,21685+0,02959 D A P+0,000501 D A P^{2}$

Em que: $\mathrm{VC}=$ volume comercial $\left(\mathrm{m}^{3}\right) ; \mathrm{DAP}$ $=$ diâmetro à altura do peito $(\mathrm{cm})$.

$\mathrm{O}$ volume comercial foi calculado usando o centro de classe de diâmetro para todas as espécies com diâmetro superior a $45 \mathrm{~cm}$ consideradas nos cálculos.

Foram definidas as estruturas por grupo de espécies, através do ajuste do método de área basal (máximo dap-q), para adequar a floresta remanescente ao sistema balanceado, visando manter a regularidade proporcional de árvores nas classes diamétricas. As equações das distribuições, por grupo de espécies, foram ajustadas pela equação de Meyer (SCHNEIDER e FINGER, 2000):

$$
N_{i}=K e^{-a d_{i}}
$$


Em que: $\mathrm{N}_{\mathrm{i}}=$ frequência por classe de diâmetro; $d_{i}=$ centro de classe de diâmetro; $\mathrm{k}$ e $\mathrm{a}=$ coeficientes; $e=$ base do logaritmo neperiano.

\section{RESULTADOS E DISCUSSÃO}

Foram identificadas 26 espécies arbóreas, segundo os critérios de classificação usados pela empresa em que foi realizado o trabalho.

$\mathrm{O}$ volume comercial total encontrado para as 26 espécies, na unidade de produção, foi de $17.021,5 \mathrm{~m}^{3}$ na área da amostra $\left(31,1 \mathrm{~m}^{3} \mathrm{ha}^{-1}\right)$, tendo sido definidos três grupos de espécies com crescimento (IPAv\%) estabelecido através da diferença de incremento médio anual de $1 \%$. Os valores situaram-se abaixo de $1,9 \%$; entre 2 e $2,9 \%$, e acima de $3,0 \%$, onde se analisou cada grupo de crescimento individualmente. Os grupos foram separados por análise cluster, com o teste $\mathrm{F}$ indicando diferença estatística entre os grupos.

$\mathrm{Na}$ área em estudo foram encontradas 26 espécies arbóreas com mercado local, regional ou de exportação. Destas 26 espécies, 6 foram indentificadas até gênero e uma ficou sem identificação. As 26 espécies estão incluídas em 25 gêneros e 15 famílias botânicas (Tabela 1). Foram levantadas as árvores com DAP $\geq 45 \mathrm{~cm}$. A estrutura abaixo de $45 \mathrm{~cm}$ de DAP foi obtida através da PP.

TABELA 1: Incremento periódico anual percentual em volume para cada grupo, em um compartimento de 547 ha.

TABLE 1: Periodical annual increment, in percentage of volume for each group, in a compartment of 547 ha.

\begin{tabular}{|c|c|c|c|c|}
\hline Espécies & $\mathrm{N}$ & $\mathrm{IPAv} \%$ & $\begin{array}{c}\text { IPAv } \% \text { Médio do } \\
\text { Grupo }\end{array}$ & $\mathrm{S}$ \\
\hline Aspidosperma vargasii A. DC. & 372 & 1,00 & - & 0,069 \\
\hline Manilkara huberi (Ducke) Standl. & 344 & 1,18 & - & 0,007 \\
\hline Guarea trichilioides L. & 278 & 1,58 & - & 0,254 \\
\hline Carapa guianensis Aubl. & 1.156 & 1,62 & - & 0,10 \\
\hline Tabebuia spp. & 882 & 1,74 & - & 0,077 \\
\hline Andira sp. & 140 & 1,76 & - & - \\
\hline Pouteria echinocarpa $=$ W. A. Rodrigues & 409 & 1,98 & - & 0,049 \\
\hline Total do grupo I & 3.581 & - & 1,59 & \\
\hline Hymenaea courbaril L. & 652 & 2,15 & - & 0,09 \\
\hline Astronium lecointei Ducke & 350 & 2,38 & - & 0,239 \\
\hline Torresea acreana Ducke & 147 & 2,40 & - & 0,040 \\
\hline Terminalia amazonica (J.F.Gmel.) Excell & 172 & 2,55 & - & 0,435 \\
\hline NI & 238 & 2,60 & - & 0,050 \\
\hline Schefflera morototoni (Aubl. Maguire, Steyerm \& Frodin) & 123 & 2,60 & - & 0,050 \\
\hline Schizolobium amazonicum Huber ex Ducke & 76 & 2,60 & - & 0,013 \\
\hline Peltogyne sp. & 3.575 & 2,67 & - & 0,540 \\
\hline Vochisia vismiifolia Spruce at Warm. & 70 & 2,67 & - & 0,167 \\
\hline Castilla ulei Warb. & 518 & 2,68 & - & 0,380 \\
\hline Peltogyne sp. & 380 & 2,76 & - & 0,050 \\
\hline Copaifera langdsdorffii Desf. & 328 & 2,80 & - & 0,072 \\
\hline Apuleia leiocarpa (Vogel) J.F.Macbr & 487 & 2,96 & - & 0,050 \\
\hline Total do grupo II & 7.116 & & 2,62 & \\
\hline Protium sp. & 2.782 & 3,22 & - & 0,200 \\
\hline Dipteryx odorata (Aubl.) Willd. & 618 & 3,39 & - & 0,350 \\
\hline Parkia multijuga Benth. & 284 & 3,47 & - & 0,050 \\
\hline Cedrela odorata L. & 275 & 3,59 & - & 0,330 \\
\hline Couratari sp. & 511 & 3,74 & - & 0,380 \\
\hline Ceiba pentandra (L.) Gaerth. & 311 & 3,90 & - & 0,345 \\
\hline Total do grupo III & 4.781 & - & 3,39 & - \\
\hline
\end{tabular}

Em que: $\mathrm{N}=$ número de árvores; IPAv\% = incremento periódico anual percentual em volume; IPAv\% $\%$ incremento periódico anual percentual em volume, ponderado por grupo e por ano; $\mathrm{S}=$ desvio padrão. 
As espécies selecionadas por grupo e os incrementos periódicos anuais percentuais em volume, mediante ponderação com o número de árvores de cada espécie, para cada grupo estão indicados na Tabela 1. No grupo I, a espécie com menor IPAv percentual em volume foi a Aspidosperma vargasii A. DC. (1,00 de IPAv\%) e, a que mostrou maior IPAv\% foi a Pouteria echinocarpa, W. A. Rodrigues (1,98 de IPAv\%). O IPA percentual em volume médio ponderado, entre as espécies deste grupo, foi de $1,59 \%$. No grupo II, a espécie com menor crescimento em volume foi a Hymenaea courbaril (2,15 de IPAv\%) e a que teve maior incremento foi a Apuleia leiocarpa (Vogel) J.F.Macbr (2,96 de IPAv\%). O IPA percentual em volume médio ponderado entre as espécies do grupo II foi de 2,62\%. Pode-se verificar que a curva, na Figura 2, deste Grupo, desce mais abruptamente que a dos demais grupos, e que há um aumento de $65 \%$ do grupo II com relação ao incremento do grupo I. No grupo III, a espécie com menor crescimento em volume foi o Protium sp. (3,22 de IPAv\%) e a que mostrou maior incremento foi a Ceiba pentandra (L.) Gaerth. (3,9 de IPAv\%). O IPA percentual em volume ponderado, entre as espécies do grupo III, foi de $3,99 \%$.

Observa-se na Figura 1 que as curvas de ajuste entre IPAv\% diferiram entre os grupos, embora o padrão geral seja de redução do IPAv\%, em função do aumento do DAP.

Como pode ser observado na Figura 1, existe uma forte relação entre os centros de classe e os incrementos encontrados. A partir daí, foi possível definir intensidades de corte de 24,43\% (grupo I), $35,37 \%$ (grupo II) e $42,37 \%$ (grupo III), com possibilidade de recuperação de $100 \%$ das mesmas, ou seja, atingir o volume pré-corte. A ideia básica consiste em que a floresta possa repor o volume de corte durante o período de tempo, definido pelo ciclo de corte estabelecido, garantindo a sustentabilidade de produção da floresta (SCHNEIDER e FINGER, 2000).

Na Tabela 2 estão indicadas as taxas de corte, para cada grupo, calculadas de acordo com o volume comercial disponível. O volume comercial da unidade de produção foi de $17.021,4 \mathrm{~m}^{3} \mathrm{e}$ a taxa de corte total foi de $6.320,6 \mathrm{~m}^{3}$, ou o equivalente a $11,55 \mathrm{~m}^{3} \mathrm{ha}^{-1}$, para o grupo de interesse de 26 espécies comerciais, ou uma intensidade média de corte de $37 \%$, para um ciclo de corte de 25 anos, segundo legislação em vigor no período de avaliação. A área basal comercial foi de $2.040,59 \mathrm{~m}^{2}$ e a remanescente esperada foi de 1.408,0 $\mathrm{m}^{2}$, no compartimento de 547 ha.

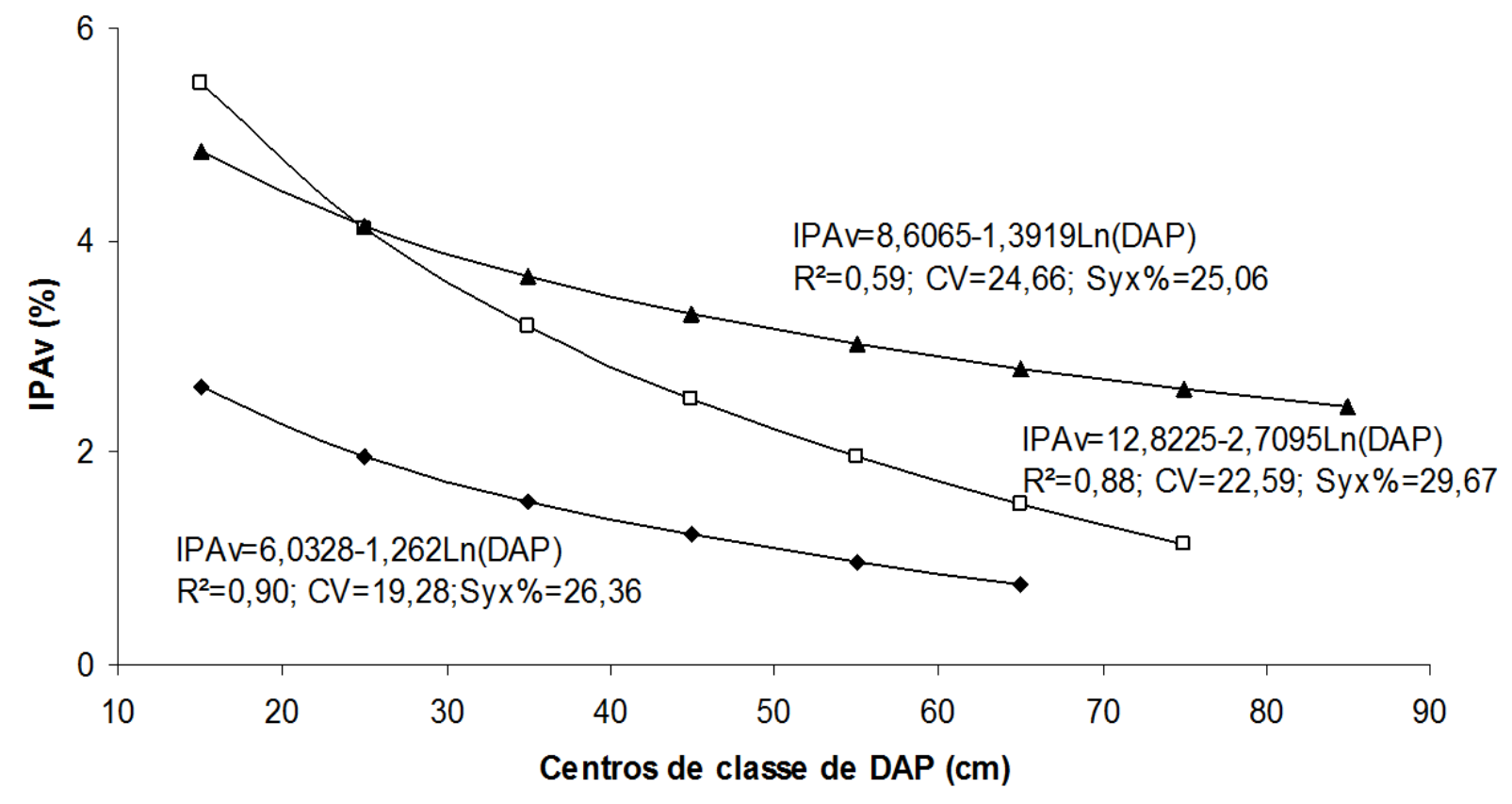

$\rightarrow-$ Grupo I $\rightarrow$-Grupo II $\rightarrow$ Grupo III

FIGURA 1: Ajuste da curva do incremento periódico anual percentual em volume relacionado ao DAP, para os grupos I, II e III.

FIGURE 1: Fitted curve of the periodical annual increment (percentual in volume) related to DBH, for groups I, II, and III. 
TABELA 2: Determinação da taxa de corte sustentada por grupo de espécies, em 547 ha.

TABLE 2: Determination of sustainable cutting rate by species group, in 547 ha.

\begin{tabular}{cccccc}
\hline Grupos & IPAv $\%$ & $\mathrm{CC}$ & $\mathrm{IC}$ & $\mathrm{VC}_{\mathrm{cc}}$ & $\mathrm{TC}$ \\
\hline I & 1,59 & 25 & 24,43 & $1.822,43$ & 445,22 \\
II & 2,62 & 25 & 35,37 & $8.063,70$ & $2.852,13$ \\
III & 3,39 & 25 & 42,37 & $7.135,33$ & $3.023,24$ \\
\hline Total & - & - & - & $17.021,46$ & $6.320,59$ \\
\hline ITC & - & - & 37,13 & - & - \\
\hline
\end{tabular}

Em que: IPAv (\%) = incremento periódico anual percentual em volume; $\mathrm{CC}=$ ciclo de corte (anos); $\mathrm{IC}=$ intensidade de corte $(\%) ; \mathrm{VC}_{\mathrm{cc}}=$ volume comercial com casca $\left(\mathrm{m}^{3}\right) ; \mathrm{TC}=$ taxa de corte $\left(\mathrm{m}^{3}\right) ; \mathrm{ITC}=$ intensidade total de corte $(\%)$.

Os diâmetros máximos desejados, para as florestas remanescentes, foram de $65 \mathrm{~cm}$ para o grupo I e $75 \mathrm{~cm}$ para os grupos II e III, de acordo com o método de área basal (máximo dap-q).

As áreas basais para os grupos I, II e III eram, respectivamente, de 240, 1061 e $939 \mathrm{~m}^{2}$. De acordo com as taxas de corte sustentáveis calculadas, as áreas basais remanescentes desejadas, a serem mantidas, foram de 181,686 e $541 \mathrm{~m}^{2}$, para os grupos I, II e III, respectivamente, e de $1.408,0 \mathrm{~m}^{2}$, no total do compartimento de 547 ha, depois de ajustadas pelo sistema máximo dap-q. O cálculo da taxa sustentável, compatibilizado ao sistema máximo dap-q, resultou em três estruturas diamétricas (Figura 2), de acordo com as restrições de taxa de corte sustentável, área basal remanescente desejada, DAP máximo, e grupo a que pertencem.
A equação de ajuste da distribuição de frequência inicial (Figura 3), por classe de diâmetro, de acordo com a exponencial negativa de Meyer, apresentou um $\mathrm{R}^{2}=0,95, \mathrm{CV}=4,88 \%$ e um Syx $=$ $0,32182\left(b_{0}=9,8106\right.$ e $\left.b_{1}=-0,04602\right)$. A equação de ajuste da distribuição de frequência remanescente (Figura 3), conjunta por classe de diâmetro obtida, teve um ajuste de $\mathrm{R}^{2}=0,93, \mathrm{CV}=6,65 \%$ e $\mathrm{Syx}=$ $0,4606\left(b_{o}=10,17567\right.$ e $\left.b_{1}=-0,05698\right)$.

Os coeficientes de Liocourt determinados foram 1,58 antes do corte e de 1,77 depois do corte, para a floresta remanescente, indicando a redução dos diâmetros maiores na estrutura final.

Também se observou que a taxa de corte conjunta (para as 26 espécies) seria de aproximadamente $37 \%$ do volume comercial disponível.

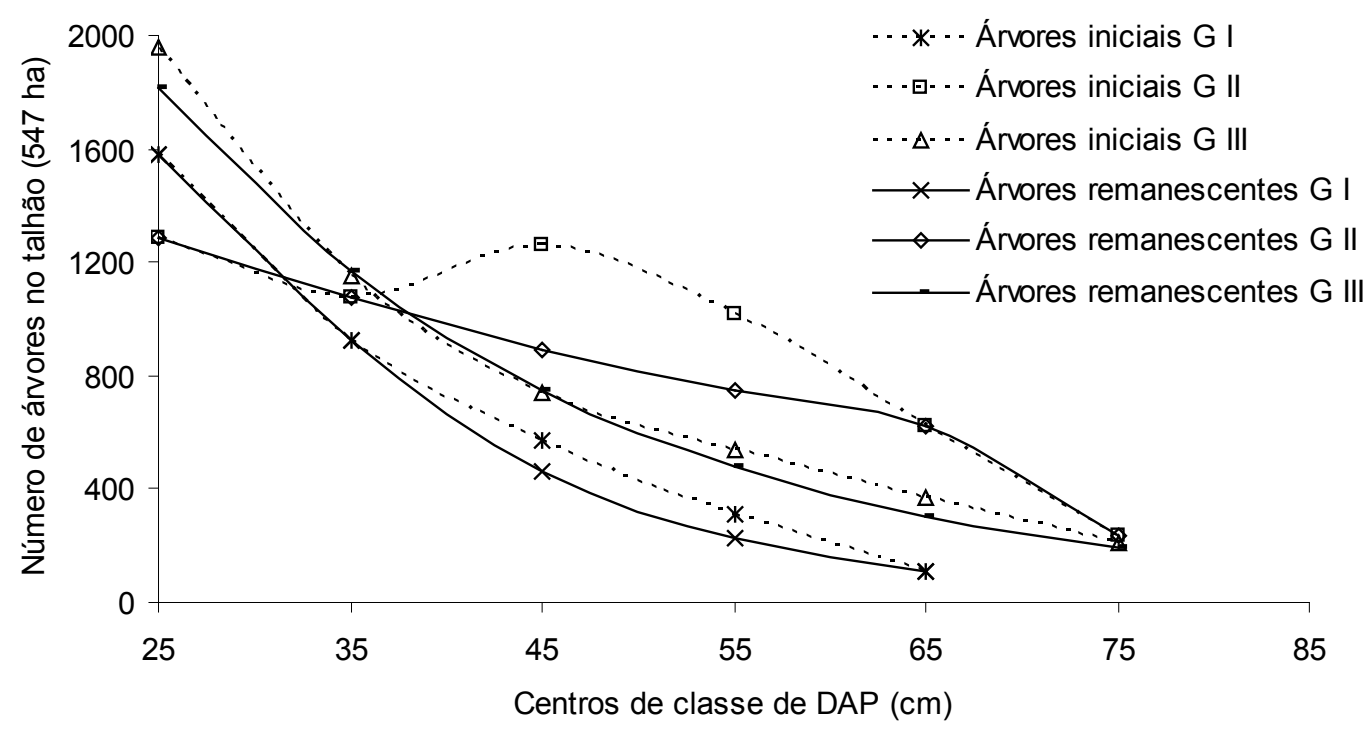

FIGURA 2: Estruturas diamétricas por grupo, antes e após simulação da taxa de corte.

FIGURE 2: Diametric structure by group, before and after allowable cut simulation. 


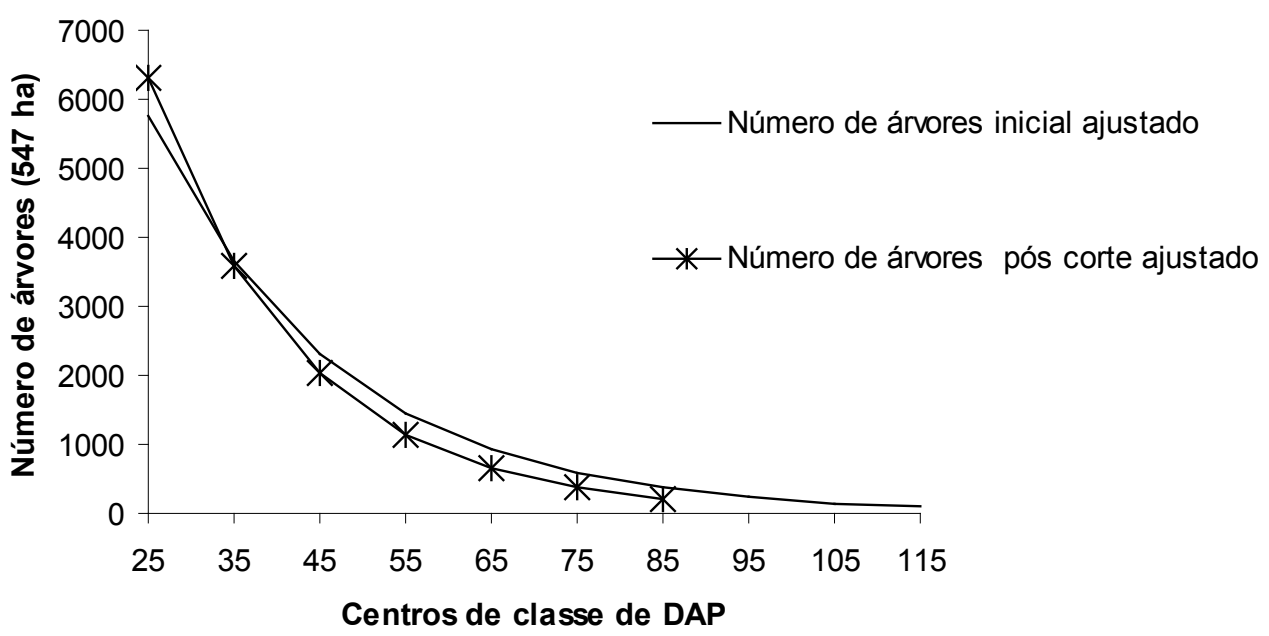

FIGURA 3: Estrutura diamétrica total antes e após simulação da taxa de corte.

FIGURE 3: Total diametric structure before and after allowable cut simulation.

Valores compatíveis foram encontrados em trabalhos que avaliaram a capacidade de recuperação da floresta e, neles são indicadas, indiretamente, as possibilidades de corte (ALDER e SILVA, 2001; AZEVEDO, 2006; VAN GARDINGEN et al., 2006). Entretanto, deve ser considerado que estes partem de uma retirada arbitrária inicial, prejudicando a aferição da capacidade de recuperação.

Ao interpretar os resultados encontrados por Alder e Silva (2001), sobre o volume que a floresta pode regenerar em ciclos de 30 anos, verifica-se que o potencial variou entre 39 e $55 \%$ do volume comercial disponível inicialmente (ao longo de uma projeção de 200 anos). Já Azevedo (2006), utilizando o modelo de simulação SYMFLOR, identificou que o incremento médio anual (IMA) deve ser, em média, 35,5\% do IMA total inicial retirado, para o ciclo de 30 anos. Outro trabalho foi desenvolvido na região de Tapajós, por Van Gardingen et al. (2006) que definiram 33\% como um limite de exploração das classes comerciais de madeira, para um ciclo de 30 anos. Sist et al. (2007) também enfatizam que retiradas mais baixas (reduções de $40-50 \%$ das retiradas atuais) são necessárias para a sustentabilidade do manejo das florestas tropicais da Amazônia.

Considerando as extrações por espécie, Schulze et al. (2008) em minucioso modelo de projeção da segunda exploração da Tabebuia, sob condições de impacto reduzido, avaliando a dinâmica da população, colonização das clareiras e taxas de recrutamento, obtiveram como limite de exploração para a espécie, $21 \%$ sob as classes comerciais. Já na proposta baseada na taxa de corte do grupo a que pertence esta espécie, as explorações seriam de 24,4\%, para um ciclo de 25 anos e, $26 \%$, para um ciclo de 30 anos. Estes são valores bastante aproximados aos obtidos nesse trabalho e confirmam que uma metodologia simples pode assegurar informações confiáveis ao manejador e órgãos fiscalizadores. Do mesmo modo, Brienen e Zuidema (2006), considerando estudo de anéis em análise prospectiva (partindo de DAPs abaixo do limite comercial) e retrospectiva (partindo de DAPs acima do limite comercial) e utilizando taxas de mortalidade, obtiveram como possibilidade de recuperação, para 4 espécies da floresta tropical amazônica estudada, valores entre 20 a 30\%, para um ciclo de 20 anos (segundo legislação da Bolívia). Sebbenn et al. (2008), em simulação utilizando o sistema Eco-Gene, obtiveram para Hymenaea, sob forte pressão de exploração (30 anos de ciclo e $45 \mathrm{~cm}$ de DAP de corte), $30 \%$ de recuperação de área basal, também semelhante ao resultado encontrado por este estudo. Assim, deve ser considerado que taxas de corte que respeitem o ritmo de crescimento das espécies serão mais uma garantia da sustentabilidade do sistema. Sabe-se que não é suficiente, para garantia de recuperação da floresta, simplesmente a retirada de baixo volume, pois se fixa as intensidades de corte, não calculadas, podem acarretar extrações baixas para espécies de rápido crescimento ou sobretaxa para espécies de crescimento lento. O estabelecimento do limite máximo de exploração em $30 \mathrm{~m}^{3} \mathrm{ha}^{-1}$ pela legislação brasileira (Instrução Normativa N. 5, 
de 11 de dezembro de 2006, na seção 1, artigo 7, parágrafo primeiro), independente da espécie que está sendo manejada, pode não ser sustentável por tratar de uma forma igual espécies com ritmos de crescimento diferentes.

\section{CONCLUSÃO}

Os planos de manejo de florestas tropicais naturais, como atualmente preconizados, não são sustentáveis, pois não consideram o real potencial de crescimento das espécies dos tipos florestais.

Os valores calculados para a taxa de corte sustentável, desta pesquisa, foram compatíveis com os trabalhos que têm avaliado a capacidade de recuperação do volume comercial das florestas tropicais.

Sugere-se o cálculo da taxa de corte, compatibilizando o incremento potencial e o ciclo de corte como balizadores da sustentabilidade do manejo, diferenciado por grupo de espécies de mesmo ritmo de crescimento.

\section{REFERENCIA BIBLIOGRÁFICA}

ALDER, D. Growth and yield of the mixed forest of the humid tropics: a review. Oxford: G. B., 1983. 44 p. (Consultancy report prepared for FAO United Nations).

ALDER, D.; SILVA, J. N. M. Sustentabilidade da produção volumétrica: um estudo de caso na Floresta Nacional de Tapajós com auxílio do modelo de crescimento CAFOGROM. In: SILVA, J. N. M.; CARVALHO, J. O. P. de; YARED, J. A. C. (Eds.). A silvicultura na Amazônia Oriental: contribuições do projeto Embrapa-DFID. Belém: Embrapa Amazônia Oriental: DFID, 2001. p.325337.

AZEVEDO, C. P. de. Dinâmica de florestas submetidas a manejo na Amazônia Oriental: experimentação e simulação. 2006. 236 f. Tese (Doutorado em Engenharia Florestal)-Universidade Federal do Paraná. Curitiba, 2006.

BORSOI, G. Subsídio para o manejo de uma floresta ombrófila mista em estágio avançado de regeneração natural. 2004. 189 f. Tese (Doutorado em Ciências Florestais)-Universidade Federal de Santa Maria. Santa Maria. 2004.

BRASIL. Instrução normativa $\mathrm{n}^{\mathrm{o}} 6$, de 23 de setembro de 2008. Diário Oficial da União, Brasília, DF, n. 185, 24 set. 2008.
Seção 1, p. 75-83.

BRAZ, E. M. Subsídios para o planejamento do manejo de florestas tropicaism da Amazônia. 2010. 236 f. Tese (Doutorado)-Universidade Federal de Santa Maria, Santa Maria, 2010.

BRIENEN, R. J. W; ZUIDEMA, P. A. The use of the tree rings in tropical forest management: Projecting timber yields of four Bolivian tree species. Forest Ecology and Management, v. 226, p. 256-267, Jan. 2006.

DAWKINS, H. C. The management of natural tropical high forest with special reference to Uganda. Oxford: Imperial Forestry Institute, 1958. 155 p. (Paper, n.. 34).

FINGER, C. A. G. Fundamentos da Biometria Florestal. Santa Maria: UFSM/CEPEF-FATEC, 1992.

OLIVEIRA, M. V. N. et al. Manejo sustentado de florestas na Amazônia Ocidental: o estudo de caso da empresa ST Manejo de Florestas. Ltda. Acta Amazônica, Manaus, v. 36, n. 3, p. 275-288, set. 2006.

PUTZ, F. E.; DYKSTRA, D. P.; HEINRICH, R. Why poor logging practices persist in the tropics. Conservation Biology, Malden, v. 14, n. 4, p. 951956, Aug. 2000.

SÁNCHEZ, M. J. S. Estudio de crecimiento y rendimiento en un bosque secundario de Costa Rica. In: SIMPOSIO INTERNACIONAL SOBRE POSIBILIDADES DE MANEJO FORESTAL SUSTENIBLE EN AMÉRICA TROPICAL, 1998, Bolívia. Memórias... Bolívia: BOLFOR, CIFOR, IUFRO, 1998. p. 241-254.

SCHNEIDER, P. R. Introdução ao manejo florestal. Santa Maria: CEPEF/FATEC, 1993. 348 p.

SCHNEIDER, P. R.; FINGER, C. A. G. Manejo Sustentado de Florestas Inequiânes Heterogêneas. Santa Maria: Universidade Federal de Santa Maria. Departamento de Ciências Florestais. 2000. 195 p. SCHULZE, M. et al. Evaluating ipê (Tabebuia, Bignoniaceae) logging in Amazonia: Sustainable management or catalyst for forest degradation? Biological Conservation, v. 141, p. 2071-2085, 2008.

SEBBENN, A. et al. Modelling the long-term impacts of selective logging on genetic diversity and demographic structure of four tropical tree species in he Amazon forest. Forest Ecology and Management, Amsterdan, v. 254. p. 335-339, 2008. SIST, P.; FERREIRA, F. N. Sustainability of reduced-impact logging in the Eastern Amazon. 
Forest Ecology and Management, Amsterdan, v. Forest, Brazil. Forest Ecology and Management, 243. p. 199-209, Feb. 2007. Amsterdan, v. 231. p. 184-195, 2006.

VAN GARDINGEN, P. R.; VALLE, VANCLAY, J. K. Modelling forest growth and D.;THOMPSON, I. Evaluation of yield regulation yield applications to mixed tropical forests. Utah: options for primary forest in Tapajo's National Walling-Ford, 1994. 304 p. 